\title{
HOMOLOGY USING CHOW VARIETIES
}

\author{
ERIC M. FRIEDLANDER
}

We introduce "Lawson homology groups" $L_{r} H_{2 r+i}\left(X, \mathbb{Z}_{l}\right)$ associated to an arbitrary projective algebraic variety $X$ over an algebraically closed field $k$ of characteristic $p \geq 0$ and a prime $l \neq p$. Our work is directly inspired by recent work of Blaine Lawson (cf. $[5,6])$, consisting in part of an algebraization of Lawson's geometric ideas and analytic arguments.

The Lawson homology group $L_{0} H_{i}\left(X, \mathbb{Z}_{l}\right)$ is the $i$ th etale $l$-adic homology group of $X$, whereas $L_{r} H_{2 r}\left(X, \mathbb{Z}_{l}\right)$ is the group of algebraic $r$-cycles on $X$ modulo algebraic equivalence (see Theorem 5 below). More generally, $L_{r} H_{2 r+i}\left(X, \mathbb{Z}_{l}\right)$ should be viewed as an $l$-adic homology group of $X$ involving " $r$ algebraic dimensions and $i$ topological dimensions." As we describe below, these groups are interesting algebraic invariants with good properties which should prove useful in the study of algebraic cycles. Moreover, the author and Barry Mazur construct maps $L_{r} H_{2 r+i}\left(X, \mathbb{Z}_{l}\right) \rightarrow$ $L_{r-1} H_{2 r+i}\left(X, \mathbb{Z}_{l}\right)$ whose iterates determine the cycle map relating algebraic cycles to etale homology.

We gratefully thank Blaine Lawson for sharing his recent results with us while still in their formative stages. We also acknowledge our great debt to Ofer Gabber whose insights were essential to our early understanding of Lawson's work. Proofs of results announced below, as well as statements and proofs of further results being developed in collaboration with Blaine Lawson and Barry Mazur, will appear elsewhere.

1. Definitions. The starting point of our work is the Chow variety $C_{r, d}(X, j)$ of effective (homogeneous, of dimension equal to) $r$ cycles of degree $d$ on the projective space $\mathbf{P}^{N}$ supported on the variety $X$, where $X$ has given a closed embedding $j: X \subset \mathbf{P}^{N}$. For example, $C_{N-1, d}\left(\mathbf{P}^{N}\right.$, id $)$ is the projective space of dimension $\left(\begin{array}{c}N+d \\ d\end{array}\right)$ whose points correspond to homogeneous forms in $N+1$ variables of degree $d$. Our Lawson homology groups $L_{r} H_{2 r+i}\left(X, \mathbb{Z}_{l}\right)$ arise by considering the group completion of the algebraic monoid $\amalg_{d \geq 0} C_{r, d}(X, j)$ of effective $r$ cycles on $X$.

We require a functor from algebraic varieties to topological spaces. We use the following composition of four functors:

$$
|\cdot|=\operatorname{Re}(\cdot) \circ \operatorname{holim}(\cdot) \circ(\mathbb{Z} / l)_{\infty}(\cdot) \circ(\cdot)_{\mathrm{et}},
$$

Received by the editors January 27, 1988.

1980 Mathematics Subject Classification (1985 Revision). Primary 14F99, 14C05; Secondary 14F20.

Partially supported by the NSF. 
where $(\cdot)_{\mathrm{et}}$ is the etale topological type functor from varieties to inverse systems of simplicial sets (cf. [4]), $(\mathbb{Z} / l)_{\infty}(\cdot)$ is the Bousfield-Kan $\mathbb{Z} / l$ completion functor (cf. [1]), holim(.) is the Bousfield-Kan homotopy inverse limit functor (cf. [1]), and $\operatorname{Re}(\cdot)$ is the geometric realization functor from simplicial sets to topological spaces.

Definition 1. Let $X$ be a projective algebraic variety with a given closed embedding $j: X \subset \mathbf{P}^{N}$, and let $r$ denote a nonnegative integer $\leq \operatorname{dim}(X)$. Applying the functor $|\cdot|$ and the bar construction to the algebraic monoid $\amalg_{d \geq 0} C_{r, d}(X, j)$, we obtain the simplicial space

$$
*=\coprod_{a \in A}\left|C_{a}\right| \equiv \coprod_{a, b \in A}\left|C_{a} \times C_{b}\right| \equiv \coprod_{a, b, c \in A}\left|C_{a} \times C_{b} \times C_{c}\right| \cdots
$$

where $A$ denotes the monoid of connected components of $\amalg_{d \geq 0} C_{r, d}(X, j)$ and $C_{a}$ denotes the component associated to $a \in A$. We denote by $\mathscr{B}_{r}(X)$ the space obtained as the geometric realization of this simplicial space and we define the stable Chow space of $r$-cycles, denoted $\Omega \mathscr{B}_{r}(X)$, to be the loop space of $\mathscr{B}_{r}(X)$.

PRoposition 2. The spaces $\mathscr{B}_{r}(X)$ and $\Omega \mathscr{B}_{r}(X)$ are infinite loop spaces. Moreover, there is a natural map of $H$-spaces $\amalg_{a \in A}\left|C_{a}\right| \rightarrow \Omega \mathscr{B}_{r}(X)$ which is a topological group completion.

If we restrict our attention to complex varieties and if we replace the functor $|\cdot|$ by the functor $(\cdot)^{\text {an }}$ which associates to a complex variety its underlying topological space with the analytic topology, then the above construction yields a space $\Omega \mathscr{B}_{r}\left(X^{\text {an }}\right)$, the analytic stable Chow space of $r$ cycles. In the special case in which $X=\mathbf{P}^{N}$, the space $\mathscr{C}_{r}\left(\mathbf{P}^{N}\right)$ considered by Lawson is homotopy equivalent to the identity component of $\Omega \mathscr{B}_{r}\left(X^{\text {an }}\right)$. More generally, $\Omega \mathscr{B}_{r}\left(X^{\text {an }}\right)$ entails the stabilization of $\amalg_{d \geq 0} C_{r, d}\left(X^{\text {an }}, j\right)$ with respect to all its connected components, whereas Lawson's original construction involved stabilization with respect to addition of multiples of a chosen linear subspace assumed to lie in $X$ [5]. Lawson's proof of his main theorem (Theorem 2 of [5]; our algebraic version is Theorem 6 below) taken in conjunction with the topological group completion property of Proposition 2 applies to prove an analogous theorem for $\Omega \mathscr{B}_{r}\left(X^{\text {an }}\right)$ valid for any complex projective variety $X$ and including a nontrivial statement concerning connected components. The homotopy groups $\pi_{*}\left(\Omega \mathscr{B}_{r}\left(X^{\text {an }}\right)\right)$ are an integral form for $\pi_{*}\left(\Omega \mathscr{B}_{r}(X)\right)$ whose complexification $\pi_{*}\left(\Omega \mathscr{B}_{r}\left(X^{\text {an }}\right)\right) \otimes \mathbb{C}$ admits the structure of a colimit of mixed Hodge structures respected by many of our algebraic arguments.

In what follows, we ignore this finer structure provided when $k=\mathbb{C}$ by $\Omega \mathscr{B}_{r}\left(X^{\text {an }}\right)$, choosing to concentrate on the more algebraic $\Omega \mathscr{B}_{r}(X)$.

Definition 3. The Lawson homology group $L_{r} H_{2 r+i}\left(X, \mathbb{Z}_{l}\right)$ is the $i$ th homotopy group of the stable Chow variety space $\Omega \mathscr{B}_{r}(X)$ for some embedding $j: X \subset \mathbf{P}^{N}$ :

$$
L_{r} H_{2 r+i}\left(X, \mathbb{Z}_{l}\right)=\pi_{i}\left(\Omega \mathscr{B}_{r}(X)\right) .
$$


2. Basic properties. In the following proposition, we state basic formal properties of the Lawson homology groups. The homotopy groups of $\Omega \mathscr{B}_{r}\left(X^{\text {an }}\right)$ also satisfy properties $4(\mathrm{a}), 4(\mathrm{c})$, and $4(\mathrm{~d})$, but not $4(\mathrm{~b})$.

Proposition 4. (a) The Lawson homology groups $L_{r} H_{2 r+i}\left(X, \mathbb{Z}_{l}\right)$ are independent (up to natural isomorphism) of the embedding $j: X \subset \mathbf{P}^{N}$ used to define them.

(b) If $X$ can be defined as the zero locus in some projective space of homogeneous equations all of whose coefficients lie in some subfield $F$ of $k$ (i.e., $X$ is defined over $F)$, then $\operatorname{Gal}(k / F)$ naturally acts on $L_{r} H_{2 r+i}\left(X, \mathbb{Z}_{l}\right)$ for any $r \leq \operatorname{dim}(X), i \geq 0$.

(c) Any map $f: X \rightarrow Y$ naturally induces a homomorphism

$$
f_{*}: L_{r} H_{2 r+i}\left(X, \mathbb{Z}_{l}\right) \rightarrow L_{r} H_{2 r+i}\left(Y, \mathbb{Z}_{l}\right)
$$

for any $0 \leq r \leq \operatorname{dim}(X), 0 \leq i$.

(d) Any flat map $g: X \rightarrow Y$ induces a homomorphism

$$
g^{*}: L_{r} H_{2 r+i}\left(Y, \mathbb{Z}_{l}\right) \rightarrow L_{r+c} H_{2 r+2 c+i}\left(X, \mathbb{Z}_{l}\right)
$$

for any $0 \leq r \leq \operatorname{dim}(Y), 0 \leq i$, where $c=\operatorname{dim}(X)-\operatorname{dim}(Y)$.

The proofs of properties 4(a), (c), (d) use rational continuous maps, our name for correspondences sufficiently like morphisms that they induce continuous maps via the functor $|\cdot|$. Property $4(\mathrm{~b})$ is a consequence of the algebraic nature of the definition of Lawson homology groups.

As mentioned earlier, the special cases $r=0$ and $i=0$ yield familiar invariants.

THeOREM 5. Assume $X$ is connected. Then

$$
L_{0} H_{i}\left(X, \mathbb{Z}_{l}\right)= \begin{cases}\mathbb{Z}, & i=0, \\ \lim H_{i}\left(X_{\mathrm{et}}, \mathbb{Z} / l^{n}\right), & i>0,\end{cases}
$$

where the inverse limit is indexed by pairs arising from the indexing category of the pro-simplicial set $X_{\mathrm{et}}$ (i.e., the etale topological type of $X$ ) and the natural numbers $n>0$. Moreover,

$$
L_{r} H_{2 r}\left(X, \mathbb{Z}_{l}\right)=(r \text {-cycles on } X) / \text { algebraic equivalence. }
$$

The proof that $L_{0} H_{i}\left(X, \mathbb{Z}_{l}\right)$ equals $l$-adic etale homology relies upon an analysis by $P$. Deligne [2] of the etale cohomology of symmetric products of varieties as well as the classical Dold-Thom theorem [3]. The fact that $L_{r} H_{2 r}\left(X, \mathbb{Z}_{l}\right)$ equals algebraic $r$-cycles modulo algebraic equivalence is proved using the group completion assertion of Proposition 2 and an understanding of the connected components of $\amalg_{d \geq 0} C_{r, d}(X, j)$.

3. Algebraic suspension. Following Lawson [5], we consider the "algebraic suspension" operation $\Sigma(\cdot)$ sending a closed algebraic subvariety $Y$ of $\mathbf{P}^{n}$ to its cone $\Sigma Y$ in $\mathbf{P}^{n+1}$ (defined by the same homogeneous equations not involving the new homogeneous coordinate). Theorem 6 is our algebraic generalization of Lawson's main theorem [5, Theorem 2]. 
THEOREM 6. Algebraic suspension $\Sigma(\cdot)$ induces a homotopy equivalence $\Sigma: \mathscr{B}_{r}(X) \rightarrow \mathscr{B}_{r+1}(\Sigma X)$. Consequently, $\Sigma$ induces isomorphisms

$$
\Sigma_{*}: L_{r} H_{2 r+i}\left(X, \mathbb{Z}_{l}\right) \rightarrow L_{r+1} H_{2 r+2+i}\left(\Sigma X, \mathbb{Z}_{l}\right)
$$

for any $0 \leq r \leq \operatorname{dim}(X), 0 \leq i$.

The proof of Theorem 6 places in the context of correspondences and Chow coordinates (cf. [7]) Lawson's proof of his suspension theorem [6]. Namely, we consider the open subset $T_{r+1, d}(\Sigma X)$ of $C_{r+1, d}(\Sigma X)$ consisting of those cycles each component of which meets $X \subset \Sigma X$ properly. We exhibit a rational continuous map which provides a homotopy deformation retraction for the subspace $\left|\Sigma\left(C_{r, d}(X)\right)\right| \simeq C_{r, d}(X)$ inside $\left|T_{r+1, d}(\Sigma X)\right|$. Furthermore, for integers $e \gg 0$, we construct another rational continuous map which provides a homotopy relating multiplication of cycles on $\Sigma X$ by $e,\left|C_{r+1, d}(\Sigma X)\right| \rightarrow\left|C_{r+1, d e}(\Sigma X)\right|$, to a map which factors through $\left|T_{r+1, d e}(\Sigma X)\right|$ and restricts to multiplication by $e$ on $\left|T_{r+1, d}(\Sigma X)\right|$. We complete the proof by interpreting the topological group completion of $\amalg_{d \geq 0}\left|C_{r+1, d}(\Sigma X)\right|$ as an $H$-space homologically equivalent to a mapping telescope.

4. Computations. Theorems 5 and 6 easily imply the following.

COROLlary 7.

$$
L_{r} H_{2 r+i}\left(\mathbf{P}^{N}, \mathbb{Z}_{l}\right)= \begin{cases}\mathbb{Z}, & i=0, \\ \mathbb{Z}_{l}, & i=2,4, \ldots, 2 N-2 r, \\ 0, & \text { otherwise. }\end{cases}
$$

Finally, we describe the Lawson homology groups associated to codimension 1 cycles on a smooth projective algebraic variety $X$.

THEOREM 8. Let $X$ be a smooth projective algebraic variety of dimension n. Then

$$
L_{n-1} H_{2 n-2+i}\left(X, \mathbb{Z}_{l}\right)= \begin{cases}\mathrm{NS}(X), & i=0, \\ \lim H_{1}\left(\operatorname{Pic}_{0}(X)_{\mathrm{et}}, \mathbb{Z} / l^{n}\right), & i=1, \\ \mathbb{Z}_{l}, & i=2, \\ 0, & \text { otherwise, }\end{cases}
$$

where $\operatorname{NS}(X)$ denotes the Neron-Severi group of $X$ and $\operatorname{Pic}_{0}(X)$ denotes the connected component of the Picard variety of $X$.

The proof of Theorem 8 entails an investigation of the natural map from a sufficiently general component of codimension 1 cycles to $\operatorname{Pic}_{0}(X)$. This enables us to verify that the homotopy type of the fiber of the map from $\left|\Omega \mathscr{B}_{n-1}(X)\right| \rightarrow|\operatorname{Pic}(X)|$ is essentially the $l$-adic homotopy type of infinite complex projective space.

\section{REFERENCES}

1. A. K. Bousfield and D. M. Kan, Homotopy limits, completions and localizations, Lecture Notes in Math., vol. 304, Springer-Verlag, Berlin and New York, 1972.

2. P. Deligne, Cohomologie des puissances symétriques, Lecture Notes in Math., vol. 305, Springer-Verlag, Berlin and New York, 1973, pp. 371-414. 
3. A. Dold and R. Thom, Quasifaserungen und unendliche symmetrische produkte, Ann. of Math. (2) 67 (1958), 239-281.

4. E. Friedlander, Etale homotopy of simplicial schemes, Ann. of Math. Studies no. 104, Princeton Univ. Press, Princeton, N.J., 1982.

5. H. B. Lawson, The topological structure of the space of algebraic varieties, Bull. Amer. Math. Soc. (N.S.) 17 (1987), 326-330.

6. __ Algebraic cycles and homotopy theory, Ann. of Math. (to appear).

7. P. Samuel, Méthodes d'algèbre abstraite en géométrie algébrique, Springer-Verlag, Berlin and New York, 1955 (revised 1967).

Department of Mathematics, Northwestern University, Evanston, Illinois 60208 
